\title{
Lymphatic Capillaries in Aging
}

\author{
Bojana Jakic ${ }^{a, b}$ Dontscho Kerjaschki ${ }^{c}$ Georg Wick ${ }^{a}$ \\ ${ }^{a}$ Laboratory of Autoimmunity, Division of Experimental Pathophysiology and Immunology, Biocenter, Medical \\ University of Innsbruck, Innsbruck, Austria; ${ }^{b}$ Department of Immunology, Genetics and Pathology, Rudbeck \\ Laboratory, Uppsala University, Uppsala, Sweden; ' Clinical Institute of Pathology, Medical University of Vienna, \\ Vienna, Austria
}

\section{Keywords}

Lymphatic vessels · Aging · Atherosclerosis · Endothelial cells

\begin{abstract}
The lymphatic system is responsible for fluid drainage from almost every organ in the body. It sustains tissue homeostasis and is also a central part of the immune system. With the discovery of cell-specific markers and transgenic mouse models, it has become possible to gain some insight into the developmental and functional roles of lymphatic endothelial cells (LECS). Only recently, a more direct regulatory role has been assigned to LECs in their functions in immunity responses and chronic diseases. Here, we discuss the changes occurring in aged lymphatic system and the role of lymphatic capillaries in some age-related diseases and experimental animal models.

(c) 2020 The Author(s)

Published by S. Karger AG, Basel
\end{abstract}

\section{Introduction}

The lymphatic system spans nearly the entire body. Lymphatic vessels are responsible for the drainage and unidirectional flow of fluid from the interstitial space back into the blood circulation. In contrast to capillaries of the blood system, lymphatic capillaries have so-called "button-like" junctions, that form loose cell-cell contact, and lack a continuous classical basement membrane. Moreover, the cell-cell junctions are disjoined and have a unique overlapping architecture which forms flaps that cause an increase in permeability, thus allowing for passive uptake of waste products and other macromolecules from the interstitium, as well as the entry of immune cells into the lymph. In addition, capillaries lack surrounding mural cells and are anchored to the extracellular matrix with the help of filaments that also assist them in their drainage function. Lymphatic capillaries start from endothelial occluded sacs. The lymph taken up by capillaries flows into the collecting lymphatic vessels, which have tighter "zipper-like" junctions and are wrapped around with mural cells [1]. Identification of lymphatic specific markers, such as the transcription factor prospero-related homeobox protein 1 (Prox1) and the cell surface proteins, namely lymphatic vessel endothelial hyaluronan receptor 1 (LYVE-1), vascular endothelial growth factor receptor 3 (VEGFR-3), and podoplanin, have enabled a great understanding of the lymphatic system [2-7]. These studies using mouse models have also enabled mapping of the tissue-specialized properties of initial and collecting vessels in different parts of the body. For instance, initial capillaries in the skin form a three-dimensional network, whereas lymphatic vessels in the lacteals of the

\begin{tabular}{ll}
\hline karger@karger.com & (c) 2020 The Author(s) \\
Published by S. Karger AG, Basel & Karger \\
Tww.karger.com/ger & This article is licensed under the Creative Commons Attribution- \\
NonCommercial-NoDerivatives 4.0 International License (CC BY- \\
NC-ND) (http://www.karger.com/Services/OpenAccessLicense). \\
Usage and distribution for commercial purposes as well as any dis- \\
tribution of modified material requires written permission.
\end{tabular}

Bojana Jakic

Department of Immunology, Genetics and Pathology Rudbeck Laboratory, Uppsala University

Dag Hammarskjölds väg 20, SE-75237 Uppsala (Sweden)

bojana.jakic@igp.uu.se 


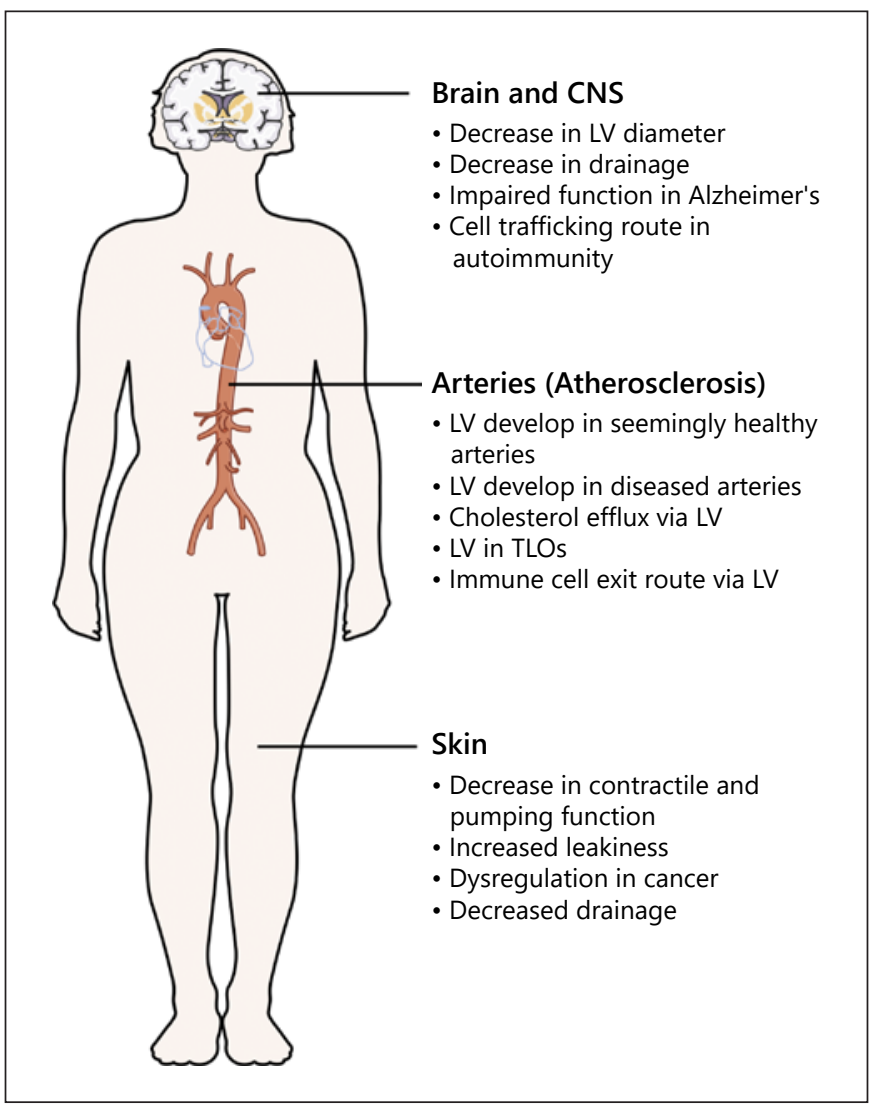

Fig. 1. Aging and age-related disease-associated changes in the lymphatic vessels. Overview of the main functions that are altered in the lymphatic vessels with aging or with diseases that are agerelated in the brain and CNS, arteries, and skin. The summary is based on references in the text. CNS, central nervous system; LV, lymphatic vessel; TLO, tertiary lymphoid organ.

gut are tube shaped that then fuse into the mesenteric collectors.

In contrast to the cardiovascular system, where circulation is powered by continuous pumping by the heart, the lymphatic system relies on either extrinsic forces such as muscle contractions from the surrounding tissues or on the intrinsic contraction by collecting vessels themselves [8-10]. Moreover, under physiological conditions, endothelial cells produce nitric oxide that maintains intrinsic pump activity $[10,11]$. Lymph that has entered the lymphatic vessels is prevented from retrograde flow by lymphatic valves. Lymphatic vessels carry the lymph through the body via lymph nodes and eventually empty the fluid into subclavian or internal jugular veins via the right lymphatic duct or the thoracic duct, and the lymph rejoins the blood $[1,12]$. On the luminal side, the lymphatic endothelial cells (LECs) are covered by proteogly- cans and glycoproteins, which are anchored to the endothelial cell membrane, and that bind to free-floating plasma proteins and endothelium-derived molecules via their side chains. This meshwork of proteoglycans and glycoproteins together with soluble components bound to their branched side chains, is collectively called the glycocalyx, and functions as a protective barrier [13]. Interestingly, the thickness and the coverage of the glycocalyx declines with age within the collecting vessels [14].

The lymphatic system is an important part of the immune system. It carries immune cells, antigens, and pathogens to draining lymph nodes. Although it was historically viewed as just a passive carrier of various components of the immune system, research in the last two decades has revealed the vast immunoregulatory roles it plays. Cancer cells take advantage of lymphatic vessels as a route to metastasize. On the other hand, a well-functioning drainage system is pivotal for a strong immune response against pathogens or any other kind of trauma that disrupts tissue homeostasis, which could lead to abnormally expanded lymphatic vessels and lymphedema. During aging, the lymphatic system undergoes considerable changes with potentially pathological consequences [14-16]. In this review, we aim to summarize how lymphatic vessels impact age-related changes in three main sites of the body which are of special interest from a gerontological perspective, namely the skin, the arteries, and the brain. There are significant age-dependent changes in the appearance and function of skin. Atherosclerosis is an age-related disorder of arteries. Furthermore, the central nervous system undergoes age-related degradation. In each section, we will discuss the physiological function of lymphatic capillaries as deduced using experimental animal models and as well as their role in the pathobiology of age-related diseases (Fig. 1).

There are of course additional important points with regard to lymphatic vessels and aging, such as the impact of smooth muscle cells and contractility, mast cells, oxidative stress, and also the diverse tissue specific roles in organs such as the thoracic duct, ovaries, prostate and eye, to mention a few. Those topics, however, are beyond the scope of this Viewpoint article, and can be found elsewhere [17].

\section{Skin}

The skin is the body's largest organ and the foremost target for external insult. Aging in the skin is manifested by loss of elasticity, wrinkles, and age spots. The mecha- 
nistic reasons underlying those changes are mainly alterations in collagenous and non-collagenous extracellular matrix components, hormonal changes, life-long accumulation of reactive oxygen species (ROS), and telomere shortening. However, some changes are induced by extrinsic forces, such as ultraviolet (UV) light, infections, and tobacco intake [18]. Intact lymphatic drainage from the skin is pivotal for the immune response against harmful agents and to sustain fluid homeostasis. Under physiological conditions, lymphatic vessels drain extracellular fluid from the skin and contribute to steady-state immune surveillance.

Lymphatic collector function generally declines with age, with for example, decreased contractile pressure and pumping frequency. On a cellular level, aged lymphatic vessels have a decrease in the glycocalyx and looser cell-to-cell gap junctions, which causes leakier vessels $[14,19]$. Skin cancer and psoriasis are two skin disorders that are aggravated in the elderly. In both cases, lymphatic vessels seem to play a major role. One study aimed to investigate the role of aged lymphatic vessels in the metastatic progress of melanoma. They found that the protein HAPLN1 (hyaluronan and proteoglycan link protein 1), that under normal physiological conditions crosslinks hyaluronan to the extracellular matrix, is reduced in aged lymphatics and contributes to increased permeability. Consequently, melanoma tumor cells were found in more distant metastatic sites in both aged melanoma patients and in aged mice [20].

In a mouse model of melanoma, tumor-infiltrating CD8 $\mathrm{T}$ cells induced expression of the inhibitory molecule programmed death-ligand 1 (PD-L1) on the surface of LECs via interferon- $\gamma($ IFN $-\gamma)$ production. PD-L1 expressed by LECs inhibited the effector function of T cells that express the receptor protein PD-1. When the same experiments were performed in mice with the LECs lacking the IFN- $\gamma \mathrm{R}, \mathrm{CD} 8$ effector function was not inhibited. This observation was also made in other skin-related pathologies, such as infection and psoriasis [21]. In another experimental setting, B16 melanoma expressing both ovalbumin (OVA) and vascular endothelial growth factor $\mathrm{C}$ (VEGF-C) was induced in mice. Although there was no difference in tumor size between melanomas expressing the foreign antigen OVA and those expressing both OVA and VEGF-C, the latter developed an enhanced lymphatic network accompanied by increased fluid drainage to draining lymph nodes. Moreover, LECs within the draining lymph nodes cross-presented the OVA peptide and inhibited CD8 effector functions [22]. In both reports, melanoma-associated lymphatic vessels were found to be beneficial for tumor survival.
The mouse model K14-VEGFR-3-Ig that lacks dermal lymphatics has proved to be useful for studying skin drainage. In one study in which trypan blue was injected intradermally or skin was painted with FITC, K14-VEGFR-3-Ig mice were found to have impaired drainage and dendritic cell migration to skin draining lymph nodes, compared to wild-type animals. Moreover, these mice displayed an altered lymph node architecture, with B cells scattered throughout the nodes as opposed to clustering into B-cell follicles as in normal lymph nodes. Moderately aged K14-VEGFR-3-Ig mice (1 year old) had increased titers of IgG1, IgG3, and IgA antibodies in the serum as well as antibody deposits in the skin. This study suggests that dermal lymphatics are important in antigen and cell drainage from the skin, and if not functioning properly, could contribute to an age-associated autoimmune phenotype [23].

As mentioned above, lymphatic drainage function declines with aging. Using luminescent dyes that were injected intradermally into the ear of young ( 2 months), middle-aged ( 7 months), and old (18 months) mice, it was shown that clearance of the dye was impaired in the older animals. Reduced drainage was directly related to fewer and smaller LYVE- $1^{+}$vessels [15]. Moreover, sitespecific delivery of VEGF-C to skin, with an engineered fusion protein where VEGF-C was fused to an antibody which recognizes an inflammation-induced component of fibronectin, expanded the lymphatic vessel area in inflamed skin. The authors used two models to induce psoriasis in mice, and in both cases, the fusion protein improved lymphatic drainage and reduced accumulation of inflammatory $\mathrm{T}$ cells [24]. In photoaged skin, the number of lymphatic vessels is reduced, and these aged vessels in addition show enhanced permeability. Exposure of a single burst of UVB to mouse skin was followed by enhanced macrophage infiltration and downregulation of VEGF-C. Edema in these mice was rescued by treatment with an intradermal injection of a mutant VEGF-C that specifically binds to VEGFR-3, and induces lymphangiogenesis [25].

Thus, as far as skin lymphatics are concerned, they could either contribute to sustain homeostasis through drainage, inhibit $\mathrm{T}$ cell responses in a melanoma setting, or allow for more distant metastases in aged skin. Therefore, it seems that dermal lymphatic vessels could play either a beneficial or harmful role. In aged skin, contractility and drainage function declines, whereas permeability increases, and collectively the homeostatic function of lymphatic capillaries and collectors decreases. 

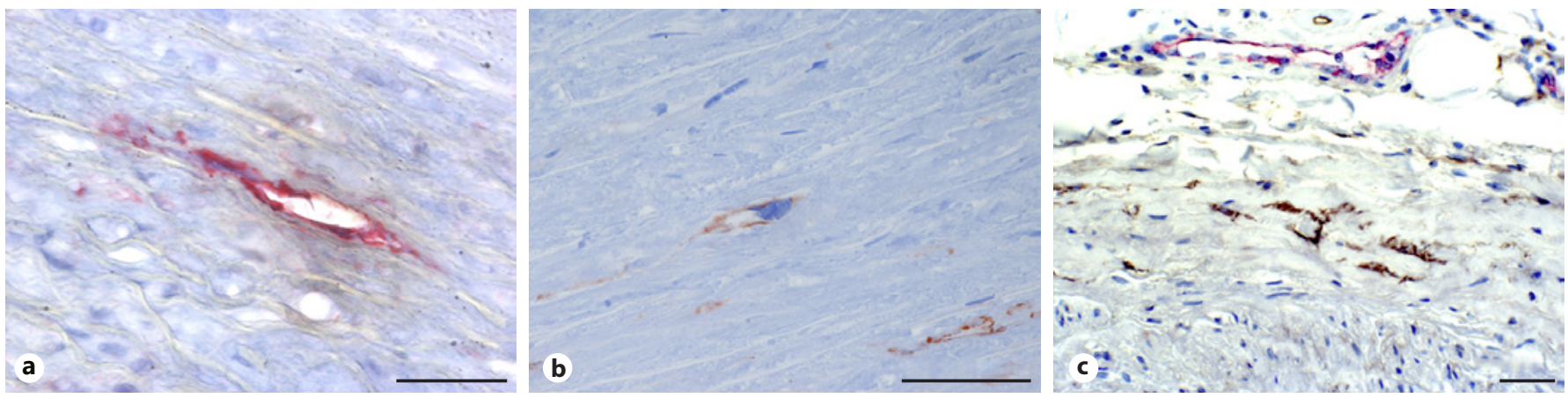

Fig. 2. Lymphatic vessels in human arteries. Formalin-fixed paraffin-embedded (FFPE) sections were de-paraffinized by xylene and re-hydrated in decreasing concentrations of ethanol. Antigens were retrieved by heat-induced bath in citrate buffer. Blocking was done with Ultra V (\#TA-060-UB, Thermo Fisher), and $2 \% \mathrm{H}_{2} \mathrm{O}_{2}$ was used to block endogenous peroxidase. UltraVision LP Large Volume Detection System AP or HRP polymer kits (TL-060-AL, TL-060-HL; Thermo Fisher) were used to detect antibody signal. Fast red, DAB (3,3'-diaminobenzidine), or AEC (3-amino-9-eth- ylcarbazole) substrate chromogens were from Sigma. a Podoplanin (clone: D2-40, homemade, D. Kerjaschki) in the media of an early atherosclerotic lesion. Scale bar $=20 \mu \mathrm{m}$. b Podoplanin (clone: D2-40) in the media of a normal human artery. Scale bar $=$ $50 \mu \mathrm{m}$. c Podoplanin-positive lymphatic vessels in brown (clone: "suzi," homemade, D. Kerjaschki) and blood vessels (CD31) in pink in the adventitia of a human atherosclerotic lesion. Scale bar $=50 \mu \mathrm{m}$.

\section{Arteries}

Arteries are large, macroscopically visible blood vessels of the body that flow into arterioles and finally capillaries. They consist of three layers: tunica intima, the innermost monolayer of endothelial cells in direct contact with the blood, tunica media, a muscular layer, and the tunica adventitia, the outermost layer attaching the vessel to the surrounding tissue. The adventitia contains both blood and lymphatic vessels under physiological conditions, with the latter necessary for sustaining homeostatic drainage. Normal function of the blood vasculature includes distribution of oxygen, nutrients, and hormones throughout the body. Moreover, immune cells, blood cells, and platelets also travel through the cardiovascular system. Arteries regulate the blood pressure through molecules within the renin-angiotensin-aldosterone system, a cross-talk mechanism between the kidneys and arteries that is in direct relation to fluid and salt intake [26]. Regular exercise, a physiological means to activate lymphatic circulation, has a beneficial impact on numerous aspects of the cardiovascular system [27].

Atherosclerosis is a multifactorial, chronic inflammatory disease of the arteries that is characterized by abnormal immune cell accumulation, lipid uptake as well as calcification and necrosis within the arterial tunica intima. In the beginning of atherogenesis, mononuclear cells, such as T cells and macrophages, enter the arterial intima via adhesion molecules which are expressed at predis- posed sites on stressed blood endothelial cells [28]. We have previously shown that autoreactive $\mathrm{T}$ cells first recognize endogenous heat shock protein 60 (HSP60) expressed on the surface of blood endothelial cells [29]. However, other autoantigens have also been proposed to drive disease, such as oxidized low-density lipoprotein and apolipoprotein B-100 [30]. Classical atherosclerosis risk factors, such as smoking, obesity, hypercholesterolemia, and low laminar blood flow, all act as endothelial cell stressors and induce HSP60 expression on the surface of endothelial cells, thus becoming a target for preexisting autoreactive $T$ cells $[28,29]$. In relation to classical risk factors and blood pressure, we have previously demonstrated that high amounts of salt can also directly stress cultured endothelial cells and cause ectopic surface HSP60 expression [31].

Although the disease starts at a young age and takes decades to develop, atherosclerosis manifests itself clinically at an older age. As the disease progresses, the arteries thicken and a nutrient and oxygen-depleted core is formed [28]. To combat this, under physiological conditions, small blood vessels, so-called vasa vasorum (Latin: the vessels of the vessels) originating from blood vessels that are found in the adventitia are formed by the angiogenic process. Blood vessels formed in plaques are often leaky, and neovascularization within the plaque is considered to contribute to unstable plaques with increased risk of rupture [32]. In addition, advanced plaques also develop lymphatic vessels within the intima and media [33]. 
Almost 50 years ago, Wolinsky and Glagov [34] showed that vasa vasorum also form in the tunica media of seemingly healthy arteries that contain 29 or more layers of smooth muscle cells. This finding, exactly reproduced in our own studies, has curiously remained unknown to the larger atherosclerosis community. Moreover, this phenomenon is age- and species-specific, as smaller species, such as the mouse, never seem to have more than five layers, whereas a human infant is already born with 34 layers of smooth muscle cells in aortas [34]. Based on the findings of Wolinsky and Glagov, we speculated that lymphatic vessels might also be present within normal arteries with $>29$ layers of smooth muscle cells. To this end, we collected normal and early lesion arterial vessels of the ascending aortae from patients undergoing surgery (unpublished results). Indeed, all samples contained more than 29 smooth muscle cell layers, with $50 \%$ having podoplanin ${ }^{+} \mathrm{CD} 31^{-}$lymphatic vessels in the media (Fig. 2).

The function of lymphatic vessels within the arterial media can only be speculated upon based on studies in mice. LEC conduits in the adventitia beneath advanced plaques in mice were shown to be essential in draining cholesterol buildup [35]. Specifically, macrophage reverse cholesterol transport, the movement of cholesterol from tissues back to the liver was impaired when aortae from $A p o E^{-/-}$mice were transplanted into donor mice that were treated with anti-VEGFR-3 antibody. The antibody treatment inhibited lymphatic vessel regrowth and in turn cholesterol uptake by LECs, leading to cholesterol buildup in the plaque [35]. Another study showed that treating $\mathrm{Ldlr}^{-/-}$mice that had diet-induced atherosclerotic plaques with VEGF-C, a factor that promotes lymphangiogenesis, stabilizes both the plaque and the drainage function of lymphatics [36]. Both aged mice and humans develop tertiary lymphoid organs (TLOs) with lymphatic vessels within the adventitia, directly adjacent to plaques $[37,38]$. In secondary lymphoid structures, such as the lymph nodes, lymphatic vessels function as a cell entry route at the afferent lymphatics and as a cell exit route at the efferent lymphatics [39]. Consequently, lymphatic vessels in disease-associated TLOs might have a similar function. In addition, LECs have been shown to directly modulate immune response by presenting antigens to both CD4 and CD8 T cells, inducing tolerance or deleting auto-reactive cells, respectively $[40,41]$. Lymph node LECs express high levels of the T cell inhibitory molecule PD-L1 [42], although to our knowledge, no such finding has yet been reported in arterial LECs. In our studies, we could not observe lymphatic vessel-associated

Aged Lymphatics mononuclear cells in the media, and therefore assign a draining function to these vessels. However, a study in mice showed that adventitial lymphatic capillaries could be a route for $\mathrm{T}$ cell exit from the plaque, in a CXCR4/ CXCL12-dependent manner [43]. Thus, experimental studies in mice and postsurgical studies in humans generally suggest that blood vessels within arterial plaques are detrimental to disease outcome, whereas lymphatic vessels seem to improve the pathophysiology of atherosclerosis [44]. Future studies are necessary to translate the findings in mouse arterial lymphatic vessels to human conditions under both physiological and inflammatory settings. Specifically, in relation to our finding that roughly half of the cohort of early lesion arteries had lymphatic vessels within the media, it would be of importance to find out if these lymphatic vasa vasorum are a prerequisite for stable plaques.

\section{The Brain and the Central Nervous System}

Although meningeal lymphatic vessels had been mentioned as early as the end of the 18th century, their existence has been largely forgotten by the scientific community. In 2015, meningeal lymphatics in the brain were rediscovered in rodents by two separate groups $[45,46]$, and later also described in the spinal cord [47], thanks to advanced imaging technology. Up until then, the brain was thought to be an organ devoid of a lymphatic drainage system. The presence of such a system in humans was confirmed 2 years later [48]. Immune cell trafficking to and from the brain was thought to occur only through the blood vasculature. Drainage was assigned to both the blood vasculature and the glymphatic system $[49,50]$. The new findings show that brain lymphatic vessels are located in the dura mater and are able to drain cerebrospinal fluid (CSF) into the deep cervical lymph nodes. They express LYVE-1, Prox1, and VEGFR-3. The studies used florescent tracers to exclude uptake by venous vessels $[45,46]$. Development of the meningeal lymphatics was shown to be dependent on VEGF-C and its receptor VEGFR-3, and in mice these were fully developed 1 month after birth [47]. Interestingly, deletion of VEGFR-3 specifically in LECs in adult mice led to a regression of the meningeal lymphatics, as well as reduced drainage of CSF. Therefore, both development and function of brain lymphatics is dependent on VEGFR-3 signaling [47].

Dural lymphatics have prompted a whole new take on age-related cognitive function and brain disorders, such 
as Alzheimer's disease. Drug-induced disruption of the brain lymphatics in a mouse model led to cognitive impairment, and a reduction in CSF and brain interstitial fluid drainage [51]. In aged mice, a decrease in the meningeal lymphatic vessel diameter and an increase in amyloid- $\beta$ accumulation in the CSF suggest the dural lymphatics as a significant route to drain amyloid- $\beta$ [51]. Another study suggested the lymphatic vessels in the brain as the main site for draining CSF, and also observed a reduction in CSF draining in aged mice [52]. However, brain fluid clearance is mainly managed by the perivascular and glymphatic system [53]. In addition to amyloid- $\beta$, both intracellular and extracellular tau have been implicated in Alzheimer's disease and other neurodegenerative diseases. In a study where mice that lack dural lymphatics were used (K14-VEGFR3-lg mice), injection of fluorescently labeled tau into the parenchyma showed significantly lower drainage from the brain compared to wildtype mice [54]. In a multiple sclerosis model, trafficking of autoreactive $\mathrm{T}$ cells through dural lymphatic vessels in a CCR7-dependent manner was directly observed [55]. Fluid uptake was first demonstrated mainly on the dorsal site $[45,46]$. This has now been expanded to also include the basal part of the skull, where the structure of meningeal lymphatic vessels seems to be different from that in the dorsal region, containing both capillary and collecting vessels with valves [56]. The authors also concluded that the basal vessels are much better in the uptake of CSF than the dorsal vessels. Lastly, decreased function of basal lymphatic vessels has been reported in aged mice [56].

Furthermore, dural lymphatics have been implicated in brain swelling (hydrocephalus), multiple sclerosis, stroke, and various neurodegenerative disorders. As the field of brain lymphatics is still young, there are more questions waiting to be answered by future studies [57].

\section{Summary}

The availability of new reagents that enable identification of LECs by various imaging methods has provided unexpected insights into the function of the lymphatic transport system in healthy young and adult animals and humans. Interestingly, there are very few studies addressing age-dependent alterations of the lymphatic system and its functional consequences. Here, we briefly review the most important findings on this topic in relation to the brain, the skin, and the vascular system. The brain is the target for a number of neurodegenerative conditions, such as Alzheimer's disease, where impaired lymphatic drainage seems to play an important pathogenetic role. The skin obviously is the organ where age-related changes are most visible and where studies of morphological and functional alterations can be readily conducted. Finally, in the field of cardiovascular research, very little work has been done on the role of the lymphatic system in the development of atherosclerosis, the most frequent age-dependent disease. Here, we present data showing the existence of lymphatic vasa vasorum not only in atherosclerotic lesions but also in the wall of normal, healthy arteries. Thus, lymphatic vessels obviously play important roles in normal and pathological aging, and it is therefore surprising that this interesting topic has so far received only scarce interest in basic and applied gerontological research.

\section{Acknowledgments}

The authors would like to thank former members of the LAI, as well as Dr. Christoph Krapf and Prof. Michael Grimm from the Department of Cardiac Surgery, Innsbruck, Austria, for making human surgical arterial specimens available to us and Christian Höpperger and Prof. Christian Ensinger from the Department of General Pathology at the Medical University Innsbruck, for their help with histological staining.

\section{Statement of Ethics}

Patients undergoing routine aortic vessel and arterial bypass surgery were recruited and the samples were collected with written informed consent, according to the regulations of the Ethics Committee of the Medical University of Innsbruck Ethics (Approval No.: UN5215 328/4.26 [3404b]).

\section{Disclosure Statement}

The authors have no conflicts of interest to disclose.

\section{Funding Sources}

This research was funded by the Austrian National Bank (Grant No. 15953) (G.W), the Science Fund of the State of Tyrol (Grant No. UNI-0404/1395) (B.J.) and the Lore-and-Udo Saldow Donation (B.J.).

\section{Author Contributions}

B.J. and G.W. wrote the first draft of the manuscript. D.K. contributed with expertise scientific advice. All authors contributed to the editing of the final manuscript. 


\section{References}

1 Swartz MA. The physiology of the lymphatic system. Adv Drug Deliv Rev. 2001 Aug;50(12):3-20.

2 Mäkinen T, Jussila L, Veikkola T, Karpanen T, Kettunen MI, Pulkkanen KJ, et al. Inhibition of lymphangiogenesis with resulting lymphedema in transgenic mice expressing soluble VEGF receptor-3. Nat Med. 2001 Feb; 7(2):199-205.

3 Petrova TV, Mäkinen T, Mäkelä TP, Saarela J, Virtanen I, Ferrell RE, et al. Lymphatic endothelial reprogramming of vascular endothelial cells by the Prox-1 homeobox transcription factor. EMBO J. 2002 Sep;21(17):4593-9.

4 Breiteneder-Geleff S, Soleiman A, Kowalski H, Horvat R, Amann G, Kriehuber E, et al. Angiosarcomas express mixed endothelial phenotypes of blood and lymphatic capillaries: podoplanin as a specific marker for lymphatic endothelium. Am J Pathol. 1999 Feb; 154(2):385-94.

5 Banerji S, Ni J, Wang SX, Clasper S, Su J, Tammi R, et al. LYVE-1, a new homologue of the CD44 glycoprotein, is a lymph-specific receptor for hyaluronan. J Cell Biol. 1999 Feb; 144(4):789-801.

6 Wigle JT, Oliver G. Prox1 function is required for the development of the murine lymphatic system. Cell. 1999 Sep;98(6):769-78.

7 Kaipainen A, Korhonen J, Mustonen T, van Hinsbergh VW, Fang GH, Dumont D, et al. Expression of the fms-like tyrosine kinase 4 gene becomes restricted to lymphatic endothelium during development. Proc Natl Acad Sci USA. 1995 Apr;92(8):3566-70.

8 McHale NG, Roddie IC. The effect of transmural pressure on pumping activity in isolated bovine lymphatic vessels. J Physiol. 1976 Oct;261(2):255-69.

9 Zawieja DC, Davis KL, Schuster R, Hinds WM, Granger HJ. Distribution, propagation, and coordination of contractile activity in lymphatics. Am J Physiol. 1993 Apr;264(4 Pt 2):H1283-91

10 Gashev AA, Davis MJ, Delp MD, Zawieja DC. Regional variations of contractile activity in isolated rat lymphatics. Microcirculation. 2004 Sep;11(6):477-92.

11 Shirasawa Y, Ikomi F, Ohhashi T. Physiological roles of endogenous nitric oxide in lymphatic pump activity of rat mesentery in vivo. Am J Physiol Gastrointest Liver Physiol. 2000 Apr;278(4):G551-6.

12 Jones D, Min W. An overview of lymphatic vessels and their emerging role in cardiovascular disease. J Cardiovasc Dis Res. 2011 Jul; 2(3):141-52.

13 Reitsma S, Slaaf DW, Vink H, van Zandvoort MA, oude Egbrink MG. The endothelial glycocalyx: composition, functions, and visualization. Pflugers Arch. 2007 Jun;454(3):34559.
14 Zolla V, Nizamutdinova IT, Scharf B, Clement CC, Maejima D, Akl T, et al. Aging-related anatomical and biochemical changes in lymphatic collectors impair lymph transport, fluid homeostasis, and pathogen clearance. Aging Cell. 2015 Aug;14(4):582-94.

15 Karaman S, Buschle D, Luciani P, Leroux JC, Detmar M, Proulx ST. Decline of lymphatic vessel density and function in murine skin during aging. Angiogenesis. 2015 Oct;18(4): 489-98.

16 Ryan T. The ageing of the blood supply and the lymphatic drainage of the skin. Micron. 2004 Apr; 35(3): 161-71. https://doi. org/10.1016/j.micron.2003.11.010.

17 Shang T, Liang J, Kapron CM, Liu J. Pathophysiology of aged lymphatic vessels. Aging. 2019 Apr; 11(16): 6602-13. https://doi. org/10.18632/aging.102213.

18 Tobin DJ. Introduction to skin aging. J Tissue Viability. 2017 Feb;26(1):37-46

19 Nagai T, Bridenbaugh EA, Gashev AA. Aging-associated alterations in contractility of rat mesenteric lymphatic vessels. Microcirculation. 2011 Aug;18(6):463-73.

20 Ecker BL, Kaur A, Douglass SM, Webster MR, Almeida FV, Marino GE, et al. Age-Related Changes in HAPLN1 Increase Lymphatic Permeability and Affect Routes of Melanoma Metastasis. Cancer Discov. 2019 Jan;9(1):82-95.

21 Lane RS, Femel J, Breazeale AP, Loo CP, Thibault G, Kaempf A, et al. IFN $\gamma$-activated dermal lymphatic vessels inhibit cytotoxic $\mathrm{T}$ cells in melanoma and inflamed skin. J Exp Med. 2018 Dec;215(12):3057-74.

22 Lund AW, Duraes FV, Hirosue S, Raghavan VR, Nembrini C, Thomas SN, et al. VEGF-C promotes immune tolerance in B16 melanomas and cross-presentation of tumor antigen by lymph node lymphatics. Cell Rep. 2012 Mar;1(3):191-9.

23 Thomas SN, Rutkowski JM, Pasquier M, Kuan EL, Alitalo K, Randolph GJ, et al. Impaired humoral immunity and tolerance in K14-VEGFR-3-Ig mice that lack dermal lymphatic drainage. J Immunol. 2012 Sep;189(5): 2181-90.

24 Schwager S, Renner S, Hemmerle T, Karaman S, Proulx ST, Fetz R, et al. Antibody-mediated delivery of VEGF-C potently reduces chronic skin inflammation. JCI Insight. $2018 \mathrm{Dec}$ 3(23):e124850. https://doi.org/10.1172/jci.insight. 124850 .

25 Kajiya K, Sawane M, Huggenberger R, Detmar M. Activation of the VEGFR-3 pathway by VEGF-C attenuates UVB-induced edema formation and skin inflammation by promoting lymphangiogenesis. J Invest Dermatol. 2009 May;129(5):1292-8.

26 Powe NR, Bibbins-Domingo K. Dietary Salt, Kidney Disease, and Cardiovascular Health. JAMA. 2016 May;315(20):2173-4.

27 Nystoriak MA, Bhatnagar A. Cardiovascular Effects and Benefits of Exercise. Front Cardiovasc Med. 2018 Sep;5:135.
28 Ross R. Atherosclerosis-an inflammatory disease. N Engl J Med. 1999 Jan;340(2):11526.

29 Wick G, Jakic B, Buszko M, Wick MC, Grundtman C. The role of heat shock proteins in atherosclerosis. Nat Rev Cardiol. 2014 Sep; 11(9):516-29.

30 Kimura T, Tse K, Sette A, Ley K. Vaccination to modulate atherosclerosis. Autoimmunity. 2015 May;48(3):152-60.

31 Jakic B, Buszko M, Cappellano G, Wick G. Elevated sodium leads to the increased expression of HSP60 and induces apoptosis in HUVECs. PLoS One. 2017 Jun;12(6):e0179383.

32 Moreno PR, Purushothaman KR, Fuster V, Echeverri D, Truszczynska H, Sharma SK, et al. Plaque neovascularization is increased in ruptured atherosclerotic lesions of human aorta: implications for plaque vulnerability. Circulation. 2004 Oct;110(14):2032-8.

33 Kholová I, Dragneva G, Cermáková P, Laidinen S, Kaskenpää N, Hazes T, et al. Lymphatic vasculature is increased in heart valves, ischaemic and inflamed hearts and in cholesterol-rich and calcified atherosclerotic lesions. Eur J Clin Invest. 2011 May;41(5):48797.

34 Wolinsky H, Glagov S. Nature of species differences in the medial distribution of aortic vasa vasorum in mammals. Circ Res. 1967 Apr;20(4):409-21.

35 Martel C, Li W, Fulp B, Platt AM, Gautier EL, Westerterp $\mathrm{M}$, et al. Lymphatic vasculature mediates macrophage reverse cholesterol transport in mice. J Clin Invest. 2013 Apr; 123(4):1571-9.

36 Milasan A, Smaani A, Martel C. Early rescue of lymphatic function limits atherosclerosis progression in Ldlr-/- mice. Atherosclerosis. 2019 Apr;283:106-19.

37 Yin C, Mohanta SK, Srikakulapu P, Weber C, Habenicht AJ. Artery Tertiary Lymphoid Organs: Powerhouses of Atherosclerosis Immunity. Front Immunol. 2016 Oct; 7:387.

38 Akhavanpoor M, Gleissner CA, Akhavanpoor H, Lasitschka F, Doesch AO, Katus HA, et al. Adventitial tertiary lymphoid organ classification in human atherosclerosis. Cardiovasc Pathol. 2018 Jan-Feb;32:8-14.

39 Hunter MC, Teijeira A, Halin C. T Cell Trafficking through Lymphatic Vessels. Front Immunol. 2016 Dec;7:613.

40 Santambrogio L, Berendam SJ, Engelhard VH. The Antigen Processing and Presentation Machinery in Lymphatic Endothelial Cells. Front Immunol. 2019 May; 10:1033.

41 Rouhani SJ, Eccles JD, Riccardi P, Peske JD, Tewalt EF, Cohen JN, et al. Roles of lymphatic endothelial cells expressing peripheral tissue antigens in CD4 T-cell tolerance induction. Nat Commun. 2015 Apr;6(1):6771. 
42 Tewalt EF, Cohen JN, Rouhani SJ, Guidi CJ, Qiao H, Fahl SP, et al. Lymphatic endothelial cells induce tolerance via PD-L1 and lack of costimulation leading to high-level PD-1 expression on CD8 T cells. Blood. 2012 Dec; 120(24):4772-82.

43 Rademakers T, van der Vorst EP, Daissormont IT, Otten JJ, Theodorou K, Theelen TL, et al. Adventitial lymphatic capillary expansion impacts on plaque $\mathrm{T}$ cell accumulation in atherosclerosis. Sci Rep. 2017 Mar;7(1): 45263.

44 Huang LH, Lavine KJ, Randolph GJ. Cardiac Lymphatic Vessels, Transport, and Healing of the Infarcted Heart. JACC Basic Transl Sci. 2017 Aug;2(4):477-83.

45 Aspelund A, Antila S, Proulx ST, Karlsen TV, Karaman S, Detmar M, et al. A dural lymphatic vascular system that drains brain interstitial fluid and macromolecules. J Exp Med. 2015 Jun;212(7):991-9.

46 Louveau A, Smirnov I, Keyes TJ, Eccles JD, Rouhani SJ, Peske JD, et al. Structural and functional features of central nervous system lymphatic vessels. Nature. 2015 Jul;523(7560): $337-41$.
47 Antila S, Karaman S, Nurmi H, Airavaara M, Voutilainen MH, Mathivet T, et al. Development and plasticity of meningeal lymphatic vessels. J Exp Med. 2017 Dec;214(12):364567.

48 Absinta M, Ha SK, Nair G, Sati P, Luciano NJ, Palisoc M, et al. Human and nonhuman primate meninges harbor lymphatic vessels that can be visualized noninvasively by MRI. eLife. 2017 Oct;6:6.

49 Louveau A, Plog BA, Antila S, Alitalo K, Nedergaard $\mathrm{M}$, Kipnis J. Understanding the functions and relationships of the glymphatic system and meningeal lymphatics. J Clin Invest. 2017 Sep;127(9):3210-9.

50 Földi $M$. The brain and the lymphatic system revisited. Lymphology. 1999 Jun;32(2):40-4.

51 Da Mesquita S, Louveau A, Vaccari A, Smirnov I, Cornelison RC, Kingsmore KM, et al. Functional aspects of meningeal lymphatics in ageing and Alzheimer's disease. Nature. 2018 Aug;560(7717):185-91.

$52 \mathrm{Ma}$ Q, Ineichen BV, Detmar M, Proulx ST. Outflow of cerebrospinal fluid is predominantly through lymphatic vessels and is reduced in aged mice. Nat Commun. 2017 Nov; 8(1): 1434. https://doi.org/10.1038/s41467017-01484-6.
53 Tarasoff-Conway JM, Carare RO, Osorio RS, Glodzik L, Butler T, Fieremans E, et al. Clearance systems in the brain-implications for Alzheimer disease. Nat Rev Neurol. 2015 Aug; 11(8):457-70.

54 Patel TK, Habimana-Griffin L, Gao X, Xu B, Achilefu S, Alitalo K, et al. Dural lymphatics regulate clearance of extracellular tau from the CNS. Mol Neurodegener. 2019 Feb; 14(1): 11.

55 Louveau A, Herz J, Alme MN, Salvador AF, Dong MQ, Viar KE, et al. CNS lymphatic drainage and neuroinflammation are regulated by meningeal lymphatic vasculature. Nat Neurosci. 2018 Oct;21(10):1380-91.

56 Ahn JH, Cho H, Kim JH, Kim SH, Ham JS, Park I, et al. Meningeal lymphatic vessels at the skull base drain cerebrospinal fluid. $\mathrm{Na}$ ture. 2019 Aug;572(7767):62-6.

57 Tamura R, Yoshida K, Toda M. Current understanding of lymphatic vessels in the central nervous system. Neurosurg Rev. 2019 Jun. https://doi.org/10.1007/s10143-019-01133-0. 